\title{
Análisis comparativo del framing mediático en agencias internacionales de noticias Oriente-Occidente. Estudio de caso: Atentado al aeropuerto de Estambul
}

\section{Comparative analysis of media framing in international news agencies east-west. Case Study: Attack at Istanbul airport}

\author{
Sabina Civila de Dios \\ Investigadora de la Universidad de Cádiz, España \\ sabicivila@gmail.com \\ Código Orcid: https://orcid.org/0000-0001-6059-9893 \\ Luis M. Romero-Rodríguez \\ Profesor e investigador de la Universidad Internacional de La Rioja, España \\ luismiguel.romero@unir.net \\ Código Orcid: https://orcid.org/0000-0003-3924-1517
}

\begin{abstract}
Resumen
Las realidades sociales son constructos discursivos, por lo que las actitudes y representaciones son el reflejo de un enfoque informativo. En este sentido, las brechas culturales y lingüísticas entre distintas civilizaciones, aunado a una construcción discursiva de naturaleza bélica, pudieren estar creando el caldo de cultivo para un enfrentamiento continuo entre Oriente y Occidente. La presente investigación analiza los diferentes encuadres retóricos de las agencias internacionales de noticias Reuters, Al Arabiya, Al Jazeera y Associated Press sobre el atentado terrorista al aeropuerto de Estambul (Turquía) el 28 de julio de 2016. Se realiza un estudio cualitativo de las figuras retóricas y los tópicos más repetidos en una muestra de 144 titulares relacionados con el atentado. El poder de los medios de comunicación en la opinión pública y construcción de la realidad generan un debate sobre cómo se redactan estas noticias y sus efectos. El objetivo es comparar los encuadres entre las agencias de ambas civilizaciones e identificar los estereotipos proyectados y si se contribuye a la espectacularización del conflicto. Se obtiene como resultado principal que no hay diferencias discursivas significativas, lo que lleva a concluir que tanto en los medios y agencias internacionales de Oriente medio como de Occidente se utilizan figuras retóricas para producir cierto efecto en la población, entre las que resaltan los eufemismos, disfemismos, la demonización y la polarización discursiva, recursos que sirven para enfatizar el miedo y crear aún mayores brechas de significación social.
\end{abstract}

Forma sugerida de citar: Civila de Dios, Sabina, \& Romero-Rodríguez, Luis M. (2018). Análisis comparativo del framing mediático en agencias internacionales de noticias Oriente-Occidente. Estudio de caso: Atentado al aeropuerto de Estambul. Universitas, 29, pp. 135-156. 


\title{
Palabras clave
}

Encuadre informativo, agencias de noticias, estereotipos, discurso bélico, demonización, encuadres retóricos.

\begin{abstract}
Social realities are discursive constructs, so that attitudes and representations are the reflection of an informative approach. In this sense, the cultural and linguistic gaps between different civilizations, together with a discursive construction of a war nature, could be creating the breeding ground for a continuous confrontation between East and West. The present study analyzes the different rhetorical frames of the international news agencies Reuters, Al Arabiya, Al Jazeera and Associated Press about the terrorist attack at the Istanbul (Turkey) airport on July 28, 2016. A quantitative study of the figures speech and the most repeated topics in the headlines of the attack is carried out. The power of the media in public opinion and the construction of reality generate a discussion about how these news are spread and their effects. The objective is to compare the different rhetorical frames in both civilizations and to identify if stereotypes are projected and if this framing contributes to the spectacularization of the confict. The main result is that there are no significant discursive differences, which leads to the conclusion that east-west rhetorical figures are used to produce a certain effect in the population, among those that highlight the euphemisms, disfemisms, demonization and discursive polarization, resources that serve to emphasize fear and create even larger gaps of social significance.
\end{abstract}

\section{Keywords}

Framing, news agencies, stereotypes, war discourse, demonization, rhetorical frames.

\section{Introducción}

El 28 de junio de 2016, veintiún días más tarde de los atentados del 7 de junio, tiene lugar un atentado en el Aeropuerto Internacional Atatürk de Estambul (Turquía). Las explosiones ocurrieron en diferentes partes de la infraestructura aeroportuaria, mientras paralelamente se llevó a cabo un tiroteo en el aparcamiento y dos explosiones en la terminal de llegadas internacionales. Estos actos dejaron un total de 44 fallecidos y 239 heridos. La cobertura mediática de este hecho fue amplia y los medios de comunicación del mundo se hacían eco de las informaciones, emitiendo noticias en directo y mostrando vídeos alive del momento de los atentados. 
En las noticias sobre atentados terroristas, las cuales disponen de un contexto social e histórico que se da en un conflicto que perdura a lo largo de los años, predominan los clichés, las simplificaciones y la falta de profundidad (Hernández, 2016; Casteleiro, 2015). El discurso de los medios de comunicación construye formas concretas de entender la realidad y condiciona nuestro conocimiento compartido del mundo (Rodrigo-Alsina, 1989 y 1997). La actual presentación del Islam incluye crispaciones xenófobas y cristalizaciones identitarias, tanto en Occidente como en Oriente (Bensalah, 2006), utilizándose para ello encuadres que contienen dispositivos retóricos los cuales permiten condensar los significados (Montoya-Londoño \& MejiaVallejo, 2015). La mayoría de estos encuadres convergen en un mismo objetivo: equiparar el islam a la barbarie y mostrar aquellos que practican esta religión como seres retrasados, violentos y sanguinarios (Bensalah, 2006).

Por su parte la relación entre los medios de comunicación y el terrorismo es compleja debido a que la difusión de las acciones terroristas pueden ser propagandísticas y a su vez puede deberse a la presión que los grupos violentos ejercen sobre los medios (Torres, 2006). Por lo tanto, es imperante la necesidad de abordar los encuadres retóricos de los ataques terroristas llevados a cabo en nombre del islam para así comprender la información transmitida, identificar si se proyectan estereotipos y si se contribuye a la espectacularización a través del tratamiento informativo.

La presente investigación analizará el tratamiento informativo que las agencias internacionales de noticias, en sus plataformas de prensa digital, tuvieron con respecto al del 28 de junio de 2016 en el aeropuerto de Estambul (Turquía), a través de un análisis de contenido de base interpretativa sobre los encuadres retóricos de las principales agencias internacionales de noticias, tomando como ejes muéstrales comparativos tanto empresas informativas del mundo occidental (Reuters y AP) como del Medio Oriente (Al Jazeera y Al Arabiya). Este estudio parte de la importancia de estudiar las agencias internacionales de noticias, en el sentido que son fuentes de información replicadas -incluso a veces sin contrastar- por medios de comunicación de todo el mundo, fenómeno conocido como el "efecto ventrílocuo", en el que un solo medio se convierte en múltiples voces (Arráez, 1998). 


\section{Los medios y la creación de realidades: Estado de la cuestión}

\section{Cobertura mediática del terrorismo, framing y encuadres retóricos}

El incremento de actos terroristas alrededor del mundo se ha convertido en un problema de preocupación (Shoshani \& Slone, 2008). Entre los mecanismos primarios por los cuales esta extensión y magnificación ocurre está la guerra psicológica, que constituye el uso planificado de las comunicaciones para influir en las opiniones, emociones, actitudes y comportamientos de los grupos destinatarios (Weimann, 1983).

Las señales enviadas por los medios de comunicación a través de una cuidadosa construcción del evento terrorista está diseñada para crear y mantener la ilusión de poder más allá del ataque mismo (Hoffman \& McCormick, 2004). La cobertura de los medios de comunicación sobre las noticias de terrorismo pueden jugar un papel importante en el cambio de actitudes, tales como la percepción del enemigo y los estereotipos (Shoshani \& Slone, 2008).

Uno de los componentes más importantes de estas actitudes son, los ya nombrados anteriormente, estereotipos que describen la tendencia humana a recurrir a una imagen simplificada de un determinado grupo social frente a una compleja realidad social (Mackie \& Hamilton, 1993).

De acuerdo con Torres-Toukoumidis et al. (2017), las agencias internacionales de noticias son las que se encargan de configurar la agenda informativa, el enfoque y sus versiones, generando matrices de opinión publicas globalizadas, coincidiendo así también con el "efecto ventrílocuo" explicado por Arráez (1998), en el que pocas agencias potentes fijan el encuadre de muchos medios de comunicación.

Cualquier texto comunicativo requiere de estrategias narrativas que organicen el discurso. En el caso de los medios de comunicación, los acontecimientos noticiosos se presentan de manera sistematizada, basados en convenciones narrativas que ofrecen una explicación acerca de quién está haciendo qué, y con qué propósito -framing- (Ardèvol-Abreu, 2015). Así, el framing consiste en seleccionar algunos aspectos de la realidad y hacerlos más destacables en la comunicación (Valera, 2016). Entman (2005) explica que este "encuadre de agenda informativa" se aplica a una teoría de cascada que consta de los siguientes niveles: 
- El gobierno: se encuentra en primer lugar porque este posee más control sobre el discurso y más poder e independencia para decidir.

- Otras élites: aquellas cercanas a los gobiernos y que tienen cierta influencia en ellos.

- Medios de comunicación: se trata de un nivel complejo puesto que no todos tienen los mismos intereses ni la misma influencias, crean su propia cascada.

- Encuadres, se trata de la forma que se tiene de enviar el mensaje.

- Público, a pesar de parecer que tiene un poder mínimo, Entman (2005) no considera al público como un sujeto pasivo que acepta sin cuestionar las interpretaciones de los medios.

El abordaje del discurso a través del concepto frame pone de manifiesto la importancia de los aspectos cognitivos más allá del análisis de los elementos puramente lingüísticos que constituyen al texto (Palma \& Manrique, 2010). Desde la perspectiva de la teoría de los frames planteada por Fillmore (2006), estas estructuras conceptuales desempeñan una función protagónica como mecanismos que facilitan la organización del conocimiento del mundo y proporcionan las bases para lograr la coherencia discursiva. Lakoff (2008) considera que los frames tienen un gran poder de persuasión o manipulación puesto que mantienen un vínculo muy estrecho con el mundo emocional del receptor, cuestión que es utilizada cuando se quiere movilizar al lector (Lakoff, 2008, p. 33).

El framing $o$ encuadre contiene dispositivos retóricos que se apoyan en la organización jerárquica de los textos, así como en sus estructuras narrativas. Para el encuadre se utilizan símbolos como las figuras retóricas que permiten condensar los significados (Díaz, 2009). Como analizan Rodríguez y Castro (2012, p. 443), la mayoría de los asuntos públicos derivan en una lucha de frames, los actores buscan consolidar su poder mediante una estrategia que consiste en planificar deliberadamente la activación de determinadas asociaciones mentales, contando con un saber cultural compartido (congruencia), entonces también estamos hablando de una activación retórica.

\section{Estrategias retóricas del conflicto: Demonización, estereotipos y espectacularización}

Cada vez es más común encontrar en los medios de comunicación elementos retóricos dispuestos para la manipulación del mensaje que son emi- 
tidos por autoridades o miembros del establishment con acceso al podio discursivo social (Grijelmo, 2001). Entre estos elementos se encuentra la demonización, entendida como una técnica retorica que fija los cimientos de un enfrentamiento real o imaginario para causar temor social, buscando desinformar o alterar las percepciones y realidades sobre un acontecimiento, descripción o una persona a partir de presentarlas como nocivas para la sociedad, generalmente como pocos argumentos válidos pero con gran carga emocional (Romero-Rodríguez, Aguaded \& Gadea, 2015; Romero-Rodríguez \& Römer-Pieretti, 2016).

Por su parte, la demonización parte necesariamente de la división social -real o imaginaria- a través de la polarización, entendida como un fenómeno social que aparece cuando los individuos alinean sus creencias en posiciones extremas y en conflicto, mientras otros mantienen opiniones más moderadas o neutrales (Isenberg, 1986; Sunsteins, 2002). De acuerdo con Cañizalez (2004), la polarización implica un desconocimiento del otro y ha tenido un correlato mediático, pues también los medios y los periodistas se han atrincherado en posiciones que excluyen la posibilidad de entender al otro. La estrategia de polarización en el plano discursivo trata de minimizar los logros del oponente y de maximizar los propios, así como de minimizar los errores propios y maximizar los del otro (van Dijk, 2003, 2009).

Otra de las estrategias retóricas más comúnmente encontradas en el discurso del conflicto es el eufemismo, que etimológicamente significa "buen habla" o "habla favorable". Según Allan y Burridge (1991) un eufemismo es utilizado como alternativa a una expresión negativa, con el fin de desprestigiar u ofender a las audiencias. El eufemismo intenta aminorar los efectos de la construcción de una realidad que colisiona con los intereses o creencias de la audiencia para no afectar o reducir el daño de la percepción del emisor frente a terceros (Romero-Rodríguez, 2014).

Mientras tanto el disfemismo -que también emerge continuamente en la retórica informativa- es una forma que consiste en nombrar una realidad con una expresión peyorativa o con intención de bajarla de categoría. Este recurso suele aparecer en el discurso hegemónico no ya como instrumento de transmisión de un contenido proposicional, sino como medio de persuasión, pues se suele utilizar para ejercer control ideológico, por lo que el contenido connotativo o valorativo de las palabras elegidas suele primar sobre el valor puramente denotativo (Crespo, 2010). 
El lenguaje es un método no instintivo de comunicar ideas, emociones y deseos por medio de un sistema de símbolos producidos de manera deliberada (Sapir, 1980). El lenguaje y las diferentes formas lingüísticas son muy importantes a la hora de elaborar y construir discursivamente los hechos. Algunos pensamientos dependen del lenguaje utilizado a la hora de expresar ciertas ideas (Searle, 1997). A través del lenguaje, ocurre la espectacularización de las noticias, que de acuerdo con Cimaturibus (2011) hace referencia a la explotación exhaustiva de un acontecimiento utilizando recursos estilísticos como exageraciones o descontextualizaciones de una frase, a fin de que ésta sea más impactante a pesar que el contenido de la noticia sea superficial. Este fenómeno tiene una clara vinculación con la tendencia creciente en los medios de comunicación hacia el infoentretenimiento, es decir, a la combinación y fusión de la información con el entretenimiento y sus propias fórmulas discursivas (Lozano, 2000; Radunski, 1999).

Los medios de comunicación son los encargados de reflejar la realidad generando modelos que influyen en la creación de la identidad social (Gila \& Guil, 1999; Marín, 2012), por lo que los estereotipos surgen de los medios sociales y se aprenden a través de procesos de socialización. Los estereotipos se convierten en el instrumento de salida más cómodo a la hora de informar-pues permiten la simplificación conceptual-y establecer pautas de conductas sociales frente a las imágenes que el espectador está consumiendo, provocando la confusión de la realidad (Vega \& Martín, 1999).

\section{Materiales y método}

La presente investigación tiene como objetivo comparar el discurso periodístico de las agencias internacionales de noticias (Reuters, AP, Al Jazeera y Al Arabiya), a través de las unidades informativas relacionadas con el atentado terrorista de Atatürk de Estambul (Turquía) del 28 de junio de 2016. Se entiende como unidades informativas el compendio de contenido que integra una noticia, gracias a los cuales se logra armar el esqueleto visual de las publicaciones (Hernández, 2017). Para su consecución, se plantean como objetivos específicos: i) Comparar los encuadres retóricos (framing) entre las agencias occidentales (Reuters y Associated Press) y las agencias del mundo árabe (Al Jazeera y $\mathrm{Al}$ Arabiya); ii) Identificar si de las unidades informativas se extraen tópicos y/o estereotipos sobre una u otra civilización y; iii) Revisar 
si los medios de comunicación y en especial las agencias internacionales de noticias contribuyen a la espectacularización del conflicto a través de la polarización y demonización del mundo árabe o del mundo occidental. Como teleología, se busca demostrar si la difusión de las informaciones sobre el atentado genera tópicos e ideas relacionadas con atribuciones negativas como el miedo o estereotipos nocivos sobre una civilización en concreto.

Para dar respuesta a estas cuestiones, el estudio se abordará con diseño cualitativo y alcance exploratorio-correlacional desde un análisis de contenido de base interpretativa de los encuadres retóricos de los titulares, lo que ayudará a examinar la forma en que se elaboran los significados (Saini y Schlonsky, 2012), mediante las figuras del discurso y cómo expresan favorabilidad u oposición hacia el mundo árabe/ islam en ambas civilizaciones, así como la espectacularización. Además, se ofrece información respecto a la relación actual entre dos o más variables (correlacional), en este caso entre las agencias de comunicación occidentales (Reuters y Associated Press) y las de Medio Oriente (Al Jazeera y Al-Arabiya). Para ello se utilizará el sistema de análisis de datos cualitativos (QDA) Atlas.ti v. 8.03 para la catalogación hermenéutica de distintas muestras de titulares sobre el atentado terrorista en el Aeropuerto Internacional Atartürk de Estambul. La selección de las agencias, además de representar paridad "n-n" entre ambas civilizaciones, se justifica porque coinciden en que son las que mantuvieron más tráfico durante 2016-2017 (ver tabla 1), de acuerdo con Alexa®.

\section{Tabla 1}

Tráfico web de las agencias internacionales de noticias

\begin{tabular}{|l|l|l|l|}
\hline \multicolumn{1}{|c|}{ Agencia } & \multicolumn{1}{c|}{$\begin{array}{c}\text { Posición } \\
\text { en el ranking }\end{array}$} & \multicolumn{1}{c|}{ Web } & \multicolumn{1}{c|}{ Tráfico } \\
\hline Al Arabiya & 1985 & https://goo.gl/63dPtU & $48,80 \%$ \\
\hline Al Jazeera & 1974 & https://goo.gl/TBDnJL & $64,90 \%$ \\
\hline Reuters & 348 & https://goo.gl/NiY8cw & $63,70 \%$ \\
\hline Associated Press & 4613 & https://goo.gl/Lm9wsn & $62,00 \%$ \\
\hline
\end{tabular}

Fuente: elaboración propia a partir del ranking Alexa®

A continuación se realizó una búsqueda a través de las páginas web principales de cada una de las agencias señaladas, utilizándose como elemen- 
tos de búsqueda: Istambul/Estambul, Terrorism/Terrorismo, Attack/Ataque, Muslism/Musulman, Daesh/Isis, Jihad/ Yijadismo. Airport/ Aeropuerto, utilizando además en los algoritmos booleanos AND y OR. Posteriormente se han seleccionado todos los titulares publicados en cada una de las agencias desde el momento del atentado hasta una semana natural más tarde (del 28 de junio de 2016 al 5 de julio de 2016), obteniéndose una muestra total de 144 titulares en total (ver tabla 2 y figura 1 ).

\section{Tabla 2}

$\mathrm{N}$ de unidades informativas por agencia de noticia (del 28/06/15 al 05/07/16)

\begin{tabular}{|l|c|l|}
\hline \multicolumn{1}{|c|}{ Agencia } & Nro. unidades & \multicolumn{1}{c|}{ Página Web } \\
\hline Al Arabiya & 23 & https://goo.gl/pogvCx \\
\hline Al Jazeera & 11 & https://goo.gl/VYHP23 \\
\hline Associated Press (AP) & 36 & https://goo.gl/ceVmJ3 \\
\hline Reuters & 74 & https://goo.gl/Q23oKS \\
\hline
\end{tabular}

Fuente: elaboración propia

Figura 1

\section{Evolutivo de unidades informativas/tratamiento por día de análisis}

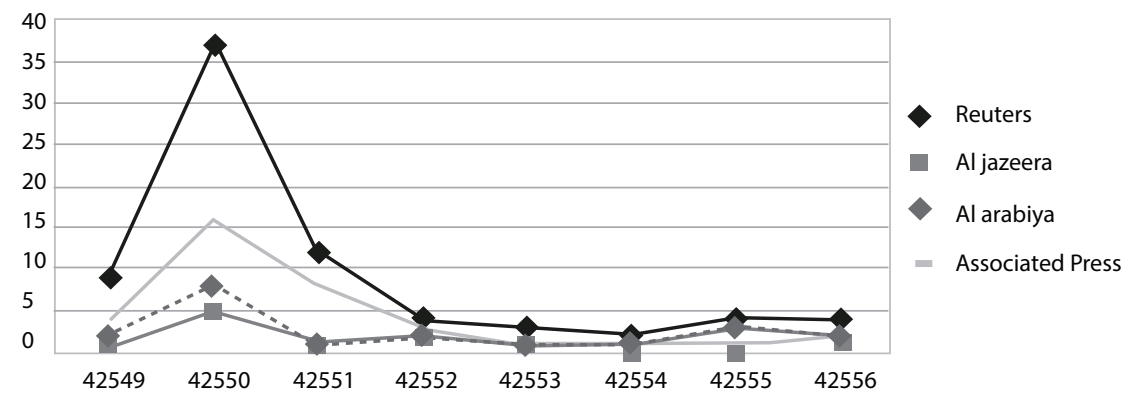

Fuente: elaboración propia

Como instrumento de análisis se utiliza la construcción de las familias discursivas emergentes utilizadas y validadas por Torres-Toukoumidis et al. (2017), que nos permitirá correlacionar y diferenciar las posiciones discur- 
sivas de las informaciones presentadas por estas agencias. Por ende, el análisis del discurso de nuestra investigación también está basado en los tres principios básicos comprendidos por van Dijk (1983): funcionalidad, significación y direccionalidad de la meta. La funcionalidad explica que el significado de las expresiones del discurso es una función de las expresiones que la componen, enfocándose en las opciones léxicas y en el estilo de las oraciones para los posibles actos que deben realizarse con la enunciación del discurso. Entonces, de acuerdo con Meersohn (2005) el significado de una oración debe ser calculado sobre la base del significado de sus palabras componentes. La significación, por su parte, se refiere al establecimiento de estructuras de coherencia en las oraciones hacia una temática general. Por último, la direccionalidad de la meta se orienta a los actos potenciales imbuidos en el discurso.

Asimismo, las familias emergentes de la ronda hermenéutica utilizadas por Torres-Toukoumidis, et al. (2017), fueron seleccionadas para el presente trabajo en virtud de la similaridad material del estudio, así como por la vinculación de las mismas con los principios comunicativos de van Dijk (1983). Estos códigos emergieron por la validación en el precitado estudio, siendo los siguientes:

\section{a. Atribución ideológica del islam}

a.1 Demonización del islam

a.2 Exaltación del islam

\section{B. Atribución ideológica del mundo árabe}

b.1 Demonización del mundo árabe

b.2 Exaltación del mundo árabe

\section{Política exterior Occidente- Medio Oriente}

c.1 Polarización Occidente-Medio Oriente

c. 2 Convivencia Occidente-Medio Oriente

D. Estado emocional deseado en la población civil

d.1 Enfatización del miedo/ pánico en la población

d.2 Enfatización de la tranquilidad/ paz de la población

E. Expresión retórica del discurso

e.1 Utilización de disfemismos

e.2 Utilización de eufemismo/ simplificación conceptual 
Los resultados obtenidos corresponden al resultado inductivo de la revisión previa de la muestra que se utiliza en su análisis, identificando la reiteración de dichos códigos de manera asistida mediante la plataforma de QDA. Para conocer los porcentajes, se realiza una regla de los resultados obtenidos en cada una de las familias emergentes.

\section{Análisis de resultados}

En el periodo analizado, correspondiente al intervalo entre el 28 de junio y el 5 de julio, las cuatro agencias presentaron un total de 144 unidades informativas. Éstas representan la muestra efectiva definitiva, ya que estaban relacionadas a los hechos y sus efectos. Reuters y AP contienen el mayor número de noticias con 74 y 36 unidades, respectivamente; mientras que $\mathrm{Al}$ Arabiya y Al Jazeera contaron con un total de 23 y 11 unidades informativas (tal como se extrae en la tabla 2). El día siguiente al atentado (29 de junio) se emitió el mayor número de unidades informativas por día (figura 1).

Del total de la muestra seleccionada se extraen un total de 286 códigos emergentes, siendo más reiterados los pertenecientes a la familia "enfatización del miedo de la población civil" con 90 codificaciones. Los "eufemismos" se sitúan en el segundo puesto más alto con un total de 75 reiteraciones. Cabe destacar que la "demonización del Islam y del mundo Árabe" cuenta con un total de 33 códigos. La "enfatización de la paz" aparece con 38 códigos emergentes, mientras que la "atribución ideológica del Islam" y la "exaltación del Islam" se sitúan en el último lugar con 13 y dos códigos.

\section{Diferencias discursivas y framing}

$\mathrm{Al}$ adaptar los criterios expuestos por el estudio semántico del discurso mediático (van Dijk, 1983) a las familias emergentes validadas y extraídas del estudio hermenéutico de las agencias internacionales de noticias realizado por Torres-Toukoumidis et al. (2017), se evidencia la siguiente vinculación:

a) Funcionalidad: La sustitución léxica es la propiedad asumida como principal indicador en este contexto específico. Si se valora la existencia de otros componentes, se observa que la reiteración de eufemismos y disfemismos acuciada por la sensibilidad del mensaje contrasta en la superficie de los discursos emitidos por las cuatro agencias internacionales (ver tabla 3 ). 


\section{Tabla 3}

\section{Sustitución léxica de las agencias internacionales en la expresión retórica del discurso}

\begin{tabular}{|l|c|c|c|c|c|}
\hline & Al Arabiya & $\begin{array}{c}\text { Al } \\
\text { Jazeera }\end{array}$ & $\begin{array}{c}\text { Associated } \\
\text { Press }\end{array}$ & Reuters & Total \\
\hline $\mathrm{N}^{\mathrm{a}}$ Eufemismos & 13 & 8 & 20 & 34 & 75 \\
\hline $\mathrm{N}^{\mathrm{a}}$ Disfemismos & 5 & 2 & 4 & 11 & 22 \\
\hline Expresión Retórica del discurso & 18 & 10 & 24 & 45 & 97 \\
\hline
\end{tabular}

Fuente: elaboración propia

Se tiene en cuenta que de los 23 titulares analizados de Al Arabiya se obtienen 13 códigos de eufemismo, por lo que se deduce que para igualarlo hipotéticamente Al Jazeera contendría 6, Reuters 42 y Associated Press 20. Por tanto se observa que las agencias internacionales de noticias que pertenecen al mundo árabe presentan un número más alto de eufemismos, aunque Associated Press lo iguala y Reuters se aproxima. Esto evidencia que se utilizan patrones reiterados basados en una manipulación del lenguaje con efectos desinformativos, por ejemplo:

\section{Eufemismo}

Turkey's Erdogan vows unity after deadly airport blasts (Al Jazeera, 29 de junio).

\section{Disfemismo}

ISIL turns 'shock and awe' doctrine against Islam (Al Jazeera, 5 de julio).

b) Significación: La figuración de la unidad del discurso sobre los atentados del 28 de junio de 2016 está representada por la coherencia local y la coherencia global manifestada en la información suscrita por las cuatro agencias internacionales. De acuerdo con Torres-Toukoumidis et al. (2017) en primer lugar la coherencia local está simbolizada por la atribución ideológica del islam y del mundo árabe, referenciando la implicación semántica del texto que derivan de los hechos, mientras que la coherencia global no es 
más que la temática general de la información expuesta en la coherencia local (ver tabla 4).

\section{Tabla 4}

Significación semántica de las codificaciones extraídas de las agencias internacionales

\begin{tabular}{|l|c|}
\hline \multicolumn{1}{|c|}{ Significación semántica } & N codificaciones \\
\hline Atribución ideológica del islam ( coherencia local) & 13 \\
\hline Atribución ideológica del mundo árabe ( coherencia local) & 31 \\
\hline Política exterior Occidente-Medio Oriente (coherencia global) & 44 \\
\hline
\end{tabular}

Fuente: elaboración propia.

Cuando se especifican las agencias internacionales y los subcódigos emergentes de la coherencia local, también existe sincronización de los datos con los subcódigos emergentes de la coherencia global. Es decir, la demonización del islam y del mundo árabe corresponde a la familia expuesta por la polarización Occidente-Medio Oriente, entretanto que la exaltación del islam y del mundo árabe corresponde a la convivencia Occidente-Medio Oriente (ver tabla 5 y 6 ).

\section{Tabla 5}

\section{Relación de códigos de connotación negativa clasificados por agencia}

\begin{tabular}{|l|l|l|l|l|l|}
\hline & $\begin{array}{c}\text { Al } \\
\text { Arabiya }\end{array}$ & $\begin{array}{c}\text { Al } \\
\text { Jazeera }\end{array}$ & $\begin{array}{c}\text { Associated } \\
\text { Press }\end{array}$ & Reuters & Total \\
\hline Demonización del islam & 2 & 2 & 3 & 4 & 11 \\
\hline Demonización del mundo árabe & 5 & 2 & 4 & 11 & 22 \\
\hline Polarización Occidente-Medio Oriente & 7 & 4 & 7 & 15 & 33 \\
\hline
\end{tabular}

Fuente: elaboración propia 


\section{Tabla 6}

\section{Relación de códigos de connotación positiva} clasificados por agencia

\begin{tabular}{|l|c|c|c|c|c|}
\hline & $\begin{array}{c}\text { Al } \\
\text { Arabiya }\end{array}$ & $\begin{array}{c}\text { Al } \\
\text { Jazeera }\end{array}$ & $\begin{array}{c}\text { Associated } \\
\text { Press }\end{array}$ & Reuters & Total \\
\hline Exaltación del islam & 1 & 1 & 0 & 0 & 2 \\
\hline Exaltación del mundo árabe & 0 & 0 & 2 & 7 & 9 \\
\hline Convivencia Occidente-Medio Oriente & 1 & 1 & 2 & 7 & 11 \\
\hline
\end{tabular}

Fuente: elaboración propia

Se evidencia en las tablas anteriores que las cuatro agencias de noticias analizadas utilizan la retórica de descrédito sobre la de reconocimiento, mientras que la demonización del mundo árabe es la atribución ideológica que mayor carga de significación semántica tiene. Asimismo se puede evidenciar con meridiana claridad que se construye un discurso inclinado hacia la polarización de Occidente-Medio Oriente, tal como se extrae de los siguientes titulares:

France temporarily closes missions in Turkey over threat (Associated Press, 5 de julio).

New York airport security increased after Istanbul attack (Associated Press, 28 de junio).

White House condemns attack on Istanbul airport (Reuters, 29 de junio).

Mientras tanto algunas noticias, mediante la polarización del mundo árabe -que tiene el mayor número de codificaciones en los aspectos positivos- equilibran la polarización con la convivencia Occidente-Medio Oriente. Este fenómeno se puede observar en los siguientes titulares:

Tunisian father who went to Turkey to bring back militant son killed (Al Arabiya, 29 de junio).

Obama: U.S. stands with Turkey after Istanbul airport attack (Reuters, 29 de junio). 
c) Direccionalidad de la meta: $\mathrm{Al}$ tomar en cuenta las familias emergentes del estudio de Torres-Toukoumidis et al. (2017), expuestas en la funcionalidad y en la significación, la finalidad comunicativa resultó asociada con las situaciones sociales proyectadas en un contexto específico (ver tabla 7).

\section{Tabla 7}

\section{Finalidad comunicativa en el estado emocional deseado a la población civil}

\begin{tabular}{|l|c|c|c|c|c|}
\hline & $\begin{array}{c}\text { Al } \\
\text { Arabiya }\end{array}$ & $\begin{array}{c}\text { Al } \\
\text { Jazeera }\end{array}$ & $\begin{array}{c}\text { Associated } \\
\text { Press }\end{array}$ & Reuters & Total \\
\hline Enfatización del miedo & 14 & 5 & 23 & 48 & 90 \\
\hline Enfatización de la tranquilidad & 8 & 5 & 8 & 17 & 38 \\
\hline $\begin{array}{l}\text { Estado emocional deseado en la } \\
\text { población civil }\end{array}$ & 22 & 10 & 31 & 65 & 128 \\
\hline
\end{tabular}

Fuente: elaboración propia

Las codificaciones extraídas demuestran cómo se expone la acentuación del miedo sobre la información dirigida a la tranquilidad y la paz en la población civil. Teniendo conocimiento de estos resultados, se observa la forma en que los medios -como replicadores del discurso de las agencias de noticias - tanto del mundo occidental como del mundo árabe recurren a la enfatización del miedo como estrategia discursiva provocando pánico. De esta forma la población acepta decisiones que no aceptaría con otros sentimientos. El uso de esta retórica se puede observar en los siguientes titulares:

German spy chief can't rule out Istanbul-style attacks at home (4 de julio en todas las agencias).

Ghostly quiet as tourists stay away from Turkey's magical Istanbul (Al Jazeera, 4 de julio).

Tips for staying safe while traveling overseas this summer (Associated Press, 29 de julio). 


\section{Estereotipos y tópicos}

Se utilizan estereotipos que acercan a Occidente el concepto de terrorismo de forma equívoca, demonizando colectivos y marginándolos al ostracismo perceptivo. En algunos titulares se estereotipa el tipo de atentado que ejerce el Estado Islámico (EI), lo que provoca que se les atribuya siempre a este grupo terrorista. Esto maximiza los rumores sobre el mundo árabe y la demonización del Islam, lo que provoca que las noticias sobre terrorismo activen el estereotipo del árabe-terrorista. Con el uso de palabras como terrorismo islámico, yihadista o extremista y resaltando las nacionalidades de los terroristas se estereotipa al mundo árabe, afiliándolo perceptivamente de forma automática al terrorismo. Se puede observar en los siguientes titulares:

CIA says Istanbul airport attack bears hallmarks of Islamic State (Reuters, 29 de junio).

US congressman: Chechen extremist behind Istanbul airport attack (Associated Press, 29 de junio).

Once again, Saudi Arabia is on the terrorism frontline (Al Arabiya, 5 de julio).

\section{Espectacularización del conflicto}

Tras la realización del análisis se ha podido observar una nueva forma de transmisión y espectacularización de los conflictos. Un atentado terrorista, como hecho noticiable, tiene todos los componentes para la espectacularización: emoción, suspenso, violencia e incertidumbre. La forma en que los medios de comunicación toman ventajas de esas características se han podido observar en los diferentes titulares en los que se encuentran:

a) Elementos multimedia, los cuales ayudan al espectador a crear una percepción de los hechos, como si de una película de ciencia ficción se tratara. Se puede observar en los siguientes titulares:

New terrifying scenes of Ataturk Airport attack (Al Arabiya, 29 de junio).

Ataturk Airport attack: Scenes of carnage in Istanbul (Al Jazeera, 29 de junio).

b) Fragmentación del contenido, lo que conlleva a que el espectador esté pendiente constantemente a las actualizaciones de los medios y a man- 
tener el interés y la incertidumbre en el conflicto. Se puede observar en los siguientes titulares:

Last hour: Obama hints at ISIS responsibilty for the Istanbul attack (Al Arabiya, 29 de junio).

ISIL 'key suspect' in Istanbul's Ataturk airport attack (Al Jazeera, 29 de junio).

c) Storytelling. Para la creación de este tipo de contenidos, de acuerdo a los titulares, se observan acciones tales como visitar familias de las víctimas y compartir historias de los testigos, lo que conecta al público de forma emocional. La forma en la que se ha utilizado el storytelling en la muestra puede ser observada en las siguientes unidades:

Tunisian father who went to Turkey to bring back militant son killed (Al Arabiya, 29 de junio).

Al Arabiya visits the family of a Saudi man killed in Istanbul attack (Al Arabiya, 30 de junio).

d) Descontextualización. El atentado de Turquía y en general, otros atentados, se han mostrado aislados de su contexto histórico y espacial, de manera que se convierten en hechos incompresibles y emocionales, lo que provoca una sensación de confusión entre la audiencia. La desinformación retiene información y la espectaculariza, haciendo noticiable aquello que vende. Se puede observar en los siguientes titulares:

Istanbul airport attack killed 2, injured 25 Saudis: envoy (Al Arabiya, 29 de junio).

Vast majority' of dead in Istanbul airport attack were Turkish, official says (Reuters, 29 de junio).

Thirty-one killed, 147 wounded in Istanbul airport attack (Reuters, 29 de junio).

41 dead in Istanbul airport attack (Associated Press, 29 de junio).

e) Titulares de intriga. Al fragmentar la información, se envían un gran número de noticias al día. Esto provoca que estemos saturados de información y que muchas no sean abiertas a la reflexión. Para que el lector abra aquellas noticias que de acuerdo al medio son relevantes, se utiliza vocabu- 
lario que deja intrigada a la audiencia como "Last hour", "special cover", "the latest". Este vocabulario capta la atención del espectador, ya que crea sensación de proporcionar actualidad y formar parte de la trama principal de la serie.

Last hour: Special coverage of Istanbul Ataturk airport attack (Al Arabiya, 29 de junio).

The Latest: Video in Turkey shows man being shot at airport (Associated Press, 30 de junio).

\section{Conclusiones y discusión}

Para alcanzar el primer objetivo específico "comparar los enfoques (framing) discursivos entre las agencias occidentales (Reuters y Associated Press) y las agencias del mundo árabe (Al Jazeera y Al Arabiya)", tras realizar el análisis, se llega a la conclusión de que no hay diferencias discursivas significativas. Todas las noticias, independientemente de la agencia a las que pertenecen, utilizan eufemismos y disfemismos, polarizan, demonizan y enfatizan el miedo. En ambos casos se utilizan figuras retóricas con el fin de producir un efecto en la población y alterar las percepciones a través del lenguaje. Llama la atención que se contabilizan un mayor número de unidades discursivas de demonización del Islam en las agencias de propiedad árabe (7 unidades), situación que puede deberse a que incluso las noticias del mundo árabe o los temas pertenecientes al mundo islámico se tratan bajo la presión de los ideales occidentales (eurocentrismo), de las relaciones con Occidente y en la medida en que Oriente se ve afectado por ellos.

También se puede observar con meridiana claridad cómo la "enfatización del miedo" es una constante en las informaciones de las cuatro agencias de noticias analizadas. Se utiliza la desinformación y descontextualización de los hechos para evitar un pensamiento crítico, omitiendo informaciones sobre la historia del conflicto y las relaciones políticas y sociales (Romero, 2012).

Asimismo y alcanzando nuestro segundo objetivo, se observa tendencia a generar estereotipos, sobre todo en las noticias del mundo occidental, lo cual no ayuda a la sensibilización de la opinión pública con el mundo árabe y produce un incremento de los prejuicios que provocan problemas en nuestro entorno social y en la posibilidad de convivencia. 
En el mismo orden de ideas y alcanzando nuestro tercer objetivo, se entrevé en los resultados que las agencias internacionales contribuyen a la espectacularización del conflicto. Esto provoca que no se garantice la profesionalidad en todas las comunicaciones, pues la calidad es menos importante que utilizar las características que imponen los canales digitales en la nueva era de la comunicación. Así también se utilizan estrategias emocionales difundiendo imágenes sensacionalistas que pueden herir la dignidad de las personas y ofreciendo representaciones distorsionadas de la realidad. En algunos casos estas estrategias se pueden considerar espectacularización positiva ya que ayudan a ampliar nuestro espacio moral, logrando sensibilizar o conmover a la audiencia frente al sufrimiento, aunque sin situar los hechos desde una mirada crítica y reflexiva.

Se afirma así que en las cuatro agencias de noticias analizadas se utilizan figuras retóricas con el fin de producir un efecto en la población y construir una realidad a través del lenguaje. En este sentido se evidencia que los medios de comunicación no dicen la manera cómo pensar pero sí en qué pensar (agenda setting) y desde qué punto abordarlo (framing). Esto se relaciona con lo referido por Tuchman (1978), que describe la noticia como una ventana cuyo marco delimita la realidad a la que se tiene acceso, limitando la percepción de otra realidad diferente y centrando la atención en ese fragmento especifico, afirmándose incluso como resultado de esta investigación que las noticias de las agencias del Medio-Oriente se tratan bajo la presión de los ideales occidentales, considerándolos como discurso predominante.

En relación a la tendencia a generar estereotipos, se observa que las agencias occidentales de noticias crean una visión negativa del Islam presentándolo como amenaza y contribuyendo a lo que se conoce como "racismo contemporáneo", el cual se basa en la autodefensa más que en las diferencias raciales. Esto confirma lo dicho por Torres-Toukoumidis et al.(2017) que afirman que los estereotipos están cobrando mayor fuerza en el plano emocional del discurso y obligan al consumidor de información a adherirse a los esquemas elaborados por los medios en base de repeticiones constantes, cuyo fin es conseguir que el espejismo se convierta en una realidad indiscutible.

Por último se observa que las noticias sobre terrorismo se han convertido en un discurso enfocado al entretenimiento de masas en ambas sociedades. Al ser noticiable y estar en la opinión pública puede crear lo que se ha denominado "espectacularización positiva", término el cual no es apoyado por Hacker (1976) que afirma que la espectacularización es innecesaria creando barreras entre el espectador de la noticia y quien lo sufre. 


\section{Referencias}

Allan, K., \& Burridge, K. (1991). Euphemism and dysphemism: language used as shield and weapon. Oxford: Oxford University Press.

Ardèvol-Abreu, E. (2015). Framing o teoría del encuadre en comunicación. Orígenes, desarrollo y panorama actual en España. Revista Latina de Comunicación Social, 70, 423-450. https://doi.org/10.4185/RLCS-2015-1053

Arráez Betancort, R. M. (1998). La comunicación para el desarrollo. Su aplicación desde el medio radio / Revisión preliminar del estado de la cuestión. Revista Latina de Comunicación Social, 9.

Bensalah, M. (2006). Islam y representaciones mediáticas. Revista CIDOB d'afers internationals, (73/74), 69-83.

Casteleiro Ruiz de Azcarate, J. (2015). Islam, terrorismo y medios de comunicación. Documento de opinión. Instituto español de estudios estratégicos, 83. Recuperado el 15 de abril de 2017 de: https://goo.gl/uDSbPu

Cañizalez, A. (2004). Apuntes sobre medios y periodistas en una sociedad polarizada. Estudios venezolanos de Comunicación, (127), 14-19.

Cimatoribus, F. (2011). La espectacularización mediática, un crecimiento desmedido. Máximo Tell. Recuperado de https://maxitell.wordpress.com/2011/12/12/ la-espectacularizacion-mediatica-un-crecimiento-desmedido/

Crespo, E. (2010). Eufemismo y disfemismo léxico en la designación del inmigrante 'sin papeles'. Bulletin of Hispanic Studies, 87(3), 273.

Entman, R. (2005). Projections of power, framing news, public opinion and U.S. Foreign Policy. Chicago: University of Chicago Press

Fillmore, C. (2006). Frame semantics. En Dirk Geerearts (Ed.), Cognitive linguistics: Basic Readings (pp. 373-401). Berlín: Mouton de Gruyter.

Gila, J., \& Guil, A. (1999). La mujer actual en los medios. Estereotipos cinematográficos. Comunicar, 12, 89-93.

Grijelmo, A. (2001). El estilo del periodista. Madrid: Taurus.

Hernández Valls, F. (2016). Terrorismo y propaganda en los medios de comunicación. El final de ETA a través de la prensa. Madrid: Universidad Complutense.

Hernández, R. (2017). Unidades informativas. Venezuela: Universidad Católica Andrés Bello.

Hoffman, B, \& McCormick, G. (2004). Terrorism, Signalling, and Suicide Attack. Studies in Conflict \& Terrorism, 27, 243-281.

Isenberg, D. (1986). Group polarization: A critical review and meta-analysis. Journal of personality social psychology, 50(6), 114. 
Lakoff, G. (2008). The political mind. New York: Penguin Group.

Lozano, J.C. (2000). Espectacularización de la información en noticieros televisivos de Canadá, Estados Unidos y México. Revista diálogo político, 12, 103-116.

Mackie, D, \& Hamilton, D. (1993). Affect, Cognition, and Stereotyping interactive Processes in Group Perception. San Diego: Academic Press.

Marín, A. L. (2012). La nueva comunicación. Madrid: Editorial Trotta, S.A

Meersohn, C. (2005). Introducción a Teun Van Dijk: Análisis de Discurso. Cinta Moebio 24, 288-302.

Montoya Londoño, C., \& Vallejo Mejías, M. (2015). Encuadres retóricos-políticos en la ley de víctimas y restitución de tierras en Colombia. Análisis político, 28(84), 23-83.

Palma, J. \& Manrique, B. (2010). La teoría de los frames en el análisis del discurso. Lingua americana, 26(14), 129 - 142.

Radunski, P. (1999). Management de la comunicación política. En J. Thesing y F. Priess (Eds.), Globalización, democracia y medios de comunicación (pp. 179-198). Buenos Aires: Centro Interdisciplinario de Estudios sobre el Desarrollo Latinoamericano (CIEDLA).

Romero, L.M. (2012). La desinformación en la nueva aldea global. Comunicación. Estudios venezolanos de la Comunicación, (159), 52-55.

Romero-Rodríguez, L. M. (2014). Pragmática de la desinformación. Estratagemas $e$ incidencia de la calidad informativa de los medios. Tesis Doctoral. Huelva: Repositorio Institucional Arias Montano. Disponible en línea en: http://rabida.uhu.es/dspace/handle/10272/9605

Romero-Rodríguez, L.M., Aguaded, I., \& Gadea, W. (2015). De la demonización a la polarización: un análisis desde el discurso digital del gobierno y la oposición venezolana. Argos, 32(62), 97-117.

Romero-Rodríguez, L. M., \& Römer Pieretti, M. (2016). Proceso de demonización de la oposición política en los hitos discursivos de Hugo Chávez según la prensa digital. Temas de Comunicación, (32).

Rodrigo Alsina, M. (1989). La construcción de la noticia. Barcelona: Paidós.

Rodrigo Alsina, M. (1997). Por un uso crítico de la prensa. Revista SIGNOS, 21.

Saini, M., \& Shlonsky, A. (2012). Systematic synthesis of qualitative research: A pocket guide for social work research methods. New York, NY: Oxford University Press.

Sapir, E. (1980). El lenguaje: introducción al estudio del habla. México: Fondo de Cultura Económico. 
Searle, J. (1997). La construcción de la realidad social. Barcelona: Universidad de Barcelona.

Shoshani, A, \& Slone, M. (2008). The Drama of Media Coverage of Terrorism: Emotional and Attitudinal Impact on the Audience. Studies in Conflict \& Terrorism, 31(7), 627-640.

Torres-Toukoumidis A., Romero-Rodríguez, L. M., Casas-Moreno, P., \& Aguaded, I. (2017). Construcción del discurso bélico desde las agencias internacionales de noticias: Estudio de caso atentados del 13 de noviembre de 2015. Revista mediterránea de comunicación, 8(1), 121-135.

Torres Romay, E. (2006). El tratamiento de la imagen en los atentados del 11-M. Terrorismo y violencia en la prensa. Tenerife: Revista Latina de Comunicación Social, 61.

Van Dijk, T. (1983). Discourse analysis: Its development and application to the structure of news. Journal of communication, 33(2), 20-43.

Van Dijk, T. (2003). Ideología y discurso. Barcelona: Ariel Gedisa.

Van Dijk, T. (2009). Discurso y poder. Barcelona: Ariel Gedisa.

Valera, L. (2016). El sesgo medio céntrico del framing en España: Una revisión crítica de la aplicación de la teoría del encuadre en los estudios de comunicación. Revista de estudios de comunicación, 41(21).

Vega, A., \& Martín, R. (1999). Los medios ante la discapacidad. Más allá de los estereotipos. Comunicar, 12, 111-116.

Fecha de recepción: 2018/04/22; Fecha de aceptación: 2018/08/10;

Fecha de publicación: 2018/09/01 